\title{
Nonalcoholic Fatty Liver Disease and Carotid Atherosclerosis in Children
}

\author{
LUCIA PACIFICO, VITO CANTISANI, PAOLO RICCI, JOHN F. OSBORN, ELISA SCHIAVO, CATERINA ANANIA, \\ EVA FERRARA, GIULIANO DVISIC, AND CLAUDIO CHIESA
}

\author{
Departments of Pediatrics [L.P., E.S., C.A., E.F.], Radiology [V.C., P.R., G.D.], and Public Health Sciences [J.F.O.], La Sapienza \\ University of Rome, 00161 Rome; National Research Council [C.C.], 00185 Rome, Italy
}

\begin{abstract}
Nonalcoholic fatty liver disease (NAFLD) is closely associated with several metabolic syndrome features, including obesity, dyslipidemia, insulin resistance, and increased cardiovascular risk. The present study was undertaken to assess whether NAFLD in children is associated with increased carotid artery intima-media thickness (IMT), a marker of early-generalized atherosclerosis. We analyzed carotid IMT along with serum triglycerides, total, lowdensity lipoprotein and high-density lipoprotein cholesterol, glucose, insulin, insulin resistance index (as homeostasis model assessment of insulin resistance), aminotransferases, leptin, and adiponectin in 29 obese children with NAFLD, 33 obese children without liver involvement, and 30 control children. The diagnosis and severity of NAFLD was based on ultrasound scan, after exclusion of infectious and metabolic disorders. Obese children with NAFLD had significantly increased carotid IMT [mean 0.58 (95\% confidence intervals $0.54-$ $0.62 \mathrm{~mm})]$ than obese children without liver involvement [0.49 $(0.46-0.52) \mathrm{mm} ; p=0.001]$ and control children $[0.40(0.36-0.43)$ $\mathrm{mm} ; p<0.0005]$. In a stepwise multiple regression model, after adjusting for age, gender, Tanner stage, and cardiovascular risk factors, the severity of fatty liver was significantly associated with maximum IMT $(b=0.08 ; p<0.0005)$. Our results suggest that NAFLD is strongly associated with carotid atherosclerosis even in childhood. (Pediatr Res 63: 423-427, 2008)
\end{abstract}

$\mathrm{N}$ onalcoholic fatty liver disease (NAFLD) encompasses a range of liver histology severity and outcomes in the absence of chronic alcohol use (1-3). The mildest form is simple steatosis in which triglycerides accumulate within hepatocytes. A more advanced form of NAFLD, nonalcoholic steatohepatitis (NASH), includes inflammation and liver cell injury. The condition was originally described in diabetic, middle-aged women without a history of significant alcohol use with liver histology consistent with alcoholic hepatitis. NAFLD is strongly associated with obesity, type 2 diabetes, and dyslipidemia, and most patients have evidence of insulin resistance $(2,3)$. Thus, NAFLD shares many features of the metabolic syndrome, a highly atherogenic condition, and its presence could signify a substantial cardiovascular risk above and beyond that conferred by individual risk factors. Although an association between hepatic steatosis and atherosclerotic risk factors has been described in adult patients (4-9), possible relationships between NAFLD and atherosclerosis in chil-

Received September 24, 2007; accepted December 7, 2007.

Correspondence: Lucia Pacifico, M.D, Department of Pediatrics, La Sapienza University of Rome, Viale Regina Elena 324, 00161 Rome, Italy; e-mail: lucia.pacifico@uniroma1.it This work was supported by a grant from La Sapienza University of Rome. dren remain to be investigated. Autoptic findings of an interesting study on the association between fatty liver and atherosclerosis in 817 children (age, 2-19 y) who died of external causes (accident, homicide, suicide) showed that fatty liver was present in $15 \%$ of the children. Mild atherosclerosis was present in $21 \%$ and moderate to severe atherosclerosis in $2 \%$. Atherosclerosis was significantly $(p<0.0001)$ more common in children with fatty liver (30\%) than those without fatty liver (19\%) (Schwimmer JB et al., Fatty liver as a determinant of atherosclerosis. Abstract presented at the 56th Annual Meeting of the American Association for the Study of Liver Diseases, November 11-15, 2005, San Francisco, CA. Hepatology 2005; 42:610). Common carotid artery intimamedia thickness (IMT) is a noninvasive marker of subclinical atherosclerotic changes and is a feasible, reliable, valid, and cost-effective method. Increased IMT is associated with cardiovascular disease (CVD) risk factors as well as the presence and severity of coronary atherosclerosis and CVD (10-14).

The aims of our study were to determine the presence of subclinical atherosclerosis in obese children with and without NAFLD and to evaluate correlates of carotid IMT in children with NAFLD. Identification of such correlates would provide a better understanding of the pathogenesis of subclinical atherosclerosis in persons with NAFLD and might lead to preventative measures.

\section{MATERIALS AND METHODS}

Subjects. Between May 2006 and April 2007, children with primary obesity [body mass index (BMI) above the 95th percentile for age and gender] who had been referred to the Department of Pediatrics, La Sapienza University of Rome, Italy, for evaluation for fatty liver upon detection of hepatomegaly (defined as a liver edge greater than $2 \mathrm{~cm}$ below the right costal margin) and/or elevated liver chemistry (defined as serum alanine aminotransferase and/or aspartate aminotransferase levels 1.5 times above normal values for age) were recruited consecutively for study participation. In these subjects, causes of chronic hepatitis other than obesity, including hepatitis B, hepatitis $\mathrm{C}$, alpha-1-antitrypsin deficiency, Wilson disease, autoimmune hepatitis, drug toxicity, and total parenteral nutrition were excluded. Children were excluded if they had type 1 or 2 diabetes, any condition known to influence body composition, insulin action, or insulin secretion (e.g., glucocorticoid therapy, hypothyroidism, Cushing disease), a history of preexisting heart disease, history of use of medications that would affect carotid IMT or lipid profile, or incomplete blood sampling. At enrollment, all patients underwent physical

Abbreviations: baPWV, brachial-ankle pulse wave velocity; BP, blood pressure; CVD, cardiovascular disease; HOMA-IR, homeostasis model assessment of insulin resistance; IMT, intima-media thickness; NAFLD, nonalcoholic fatty liver disease; NASH, nonalcoholic steatohepatitis 
examination including measurements of weight, standing height, BMI, and determination of the stage of puberty, according to Tanner criteria $(15,16)$, laboratory tests, measurements of body composition (including determinations of lean and total fat mass), liver, and carotid ultrasound.

The degree of obesity was quantified by Cole's least mean square method, which normalizes the skewed distribution of BMI and expresses BMI as a SD score (17). Measurements of body composition were made with a total body scanner (Hologic QDR-4500W, Waltham, MA) in array mode. This equipment uses a switched pulse stable dual energy x-ray operating at 100 and 140 $\mathrm{kV}$. The data were analyzed by a pediatric Hologic software version 11.2. Systolic blood pressure (BP) and diastolic BP were measured twice at the right arm after a 10-min rest in the supine position by using an automated oscillatory system (Dinamap Vital Signs Monitor, Model 1846 SX; Criticon Incorporated, Tampa, FL).

The diagnosis of NAFLD was based on ultrasound scan. The liver ultrasonographic evaluation used an Aplio XV (Toshiba America Medical Systems, Tustin, CA). Ultrasonographically, steatosis was defined by an appearance of hyperechoic liver parenchyma with tightly packed fine echoes and posterior beam attenuation. The severity of hepatic steatosis was graded as mild, moderate, or severe using the criteria described previously (18).

Over the same study period, healthy children with BMI appropriate for gender and age, attending checkups at the Department of Pediatrics, La Sapienza University of Rome, Italy, were consecutively recruited to the study if they had 1) normal aminotransferase levels; 2) normal liver ultrasound; 3) negative serology for hepatitis B and C; and 4) parents' consent to participate in the study which included anthropometric measurements, laboratory investigations, and carotid ultrasound. A positive family history of diabetes or CVD (coronary artery disease or stroke) was not an exclusionary criterion for either obese or control children.

The study was approved by the Hospital Ethics Committee, and informed consent was obtained from subjects' parents before assessment.

Laboratory investigations. Blood samples were taken from each subject, after an overnight fast, for estimation of serum concentrations of glucose, insulin, aminotransferases, total, low-density lipoprotein (LDL) and highdensity lipoprotein (HDL) cholesterol, triglycerides, leptin, and adiponectin. A RIA was used to measure human leptin (DRG Diagnostica, Marburg, Germany; detection limit, $0.5 \mathrm{ng} / \mathrm{mL}$; inter- and intra-assay CVs, 3.0-6.2\% and 3.4-8.3\%, respectively), adiponectin (DRG Diagnostica, Marburg, Germany; detection limit, $1 \mathrm{ng} / \mathrm{mL}$; inter- and intra-assay CVs, 6.9-9.2\% and $1.8-6.2 \%$, respectively), and insulin (CIS Bio International, Schering S.A., Gif-Sur-Yvette Cedex, France). The degree of insulin resistance was determined by a homeostasis model assessment of insulin resistance (HOMA-IR). Scores were calculated as the product of the fasting serum insulin level $(\mu \mathrm{U} / \mathrm{mL})$ and the fasting serum glucose level $(\mathrm{mmol} / \mathrm{L})$, divided by 22.5 .

Carotid artery studies. The same sonographer (V.C.), who was blinded to the participant's laboratory values and risk factors, did all the examinations. High-resolution B-mode ultrasonography of the common carotid arteries was performed with an Aplio XV (Toshiba America Medical Systems, Tustin, $\mathrm{CA})$ with a linear 14-MHz linear transducer, following a standardized protocol. The participants were examined in the supine position with the head turned slightly to the left. On a longitudinal, two-dimensional ultrasound image of the carotid artery, the posterior (far) wall of the carotid artery was displayed as two bright white lines separated by a hypoechogenic space. The distance between the leading edge of the first bright line of the far wall and the leading edge of the second bright line indicated the carotid artery IMT. The measurement was performed at the common carotid artery near the bifurcation during end diastole. We measured four values on each side, and the maximum and mean IMT were calculated separately for each side. Repeated measurements on the same subjects [that were done in 62 children (42 obese patients and 20 controls) randomly selected from the 92 study children] gave coefficients of variation less than $3 \%$.

Statistical analysis. Statistical analyses were performed using the SPSS package. Data are expressed either as frequencies or as means with $95 \%$ confidence intervals (CI). The measured serum triglycerides, total, HDL and LDL cholesterol, insulin, HOMA-IR, leptin, adiponectin, and IMT values were distributed with a long tail to the right (positive skew), but their logarithms were approximately normally distributed. Thus, their mean values with $95 \%$ CI are reported as geometric means. Differences between groups were tested for significance using unpaired $t$ test (two-tailed). The $\chi^{2}$ test and Fisher exact test were used, when appropriate, to compare prevalences. Pearson correlation and linear regression analysis were used to examine the relationship between carotid log IMT and independent variables. Relationships between carotid IMT and NAFLD severity score were also examined after adjustment for age, gender, Tanner stage, BMI, fat mass, systolic and diastolic BP, total, LDL and HDL cholesterol, triglycerides, HOMA-IR, leptin, and adiponectin using stepwise multiple linear regression analysis. We took the maximum value of carotid IMT as the dependent variable in the regression models because the strongest association between the different measurements of IMT and coronary risk factors in otherwise healthy individuals is achieved by applying the maximum value of IMT and not the mean value of IMT (19). A $p$ value of less than 0.05 was considered to be statistically significant.

\section{RESULTS}

Clinical and laboratory characteristics for the 62 obese children, and 30 healthy controls are summarized in Table 1 . The subjects were all independent members of the study group. No subject was included more than once and there was no family connection between the individuals studied.

The obesity and control groups showed no significant differences in terms of age, gender, and Tanner stage. They also were similar in terms of family history of CVD (14.5\% vs. $13.3 \%)$ and diabetes $(4.8 \%$ vs. $3.3 \%)$. Subjects in the obese group had a significantly higher systolic and diastolic BP. Geometric mean values of triglycerides, leptin, insulin, and HOMA-IR were significantly elevated in obese children, whereas HDL cholesterol and adiponectin values were lower than for the controls. Compared with controls, obese children showed increased mean [0.49 (95\% CI, 0.46-0.52) vs. 0.39 (95\% CI, 0.35-0.43) $\mathrm{mm} ; p<0.0005]$ and maximum [0.53 (95\% CI, 0.50-0.56) vs. 0.40 (95\% CI, 0.36-0.43) mm; $p<$ 0.0005] carotid IMT (Table 1).

In total, 29 children $(46.7 \%)$ had, on ultrasound scanning, evidence of fatty liver, 7 (24.1\%), 13 (44.8\%), and 9 (31.0\%) were found to have mild, moderate, and severe degrees of fatty liver, respectively. Twenty-two (35.5\%) of the 62 obese children had elevated serum aminotransferases. All of them had sonographic evidence of moderate to severe degrees of fatty liver indicating a presumptive diagnosis of NASH. None of the obese children with mild severity of hepatic steatosis, as well as those with no ultrasound hepatic steatosis, had elevated aminotransferases. Children with hepatic steatosis had higher values for systolic BP, higher insulin, and HOMA-IR values, and lower adiponectin concentrations than subjects with no liver involvement. Mean as well as maximum carotid IMT measurements also were higher in patients with liver steatosis (Table 1). Age, gender, Tanner stage, BMI-SD score, fat mass, lipid profile as well as leptin levels did not differ between the two patient groups.

Within the entire group of obese children, risk variables that were associated with maximum IMT in a univariate analysis were age $(p=0.019)$, Tanner stage $(p=0.001)$, BMI $(p=$ $0.004)$, fat mass $(p=0.005)$, systolic $\operatorname{BP}(p=0.009)$, alanine aminotransferase $(p=0.003)$, insulin $(p=0.01)$, HOMA-IR $(p=0.001)$, leptin $(p=0.028)$, and adiponectin $(p=0.016)$. The fatty liver severity score was also significantly associated with increased IMT $(p<0.0005)$. Gender, BMI-SD score, HDL and LDL cholesterol, and triglyceride concentrations did not significantly correlate to carotid IMT (Table 2).

The relationship between log IMT and fatty liver severity score was investigated initially by defining three dummy variables to identify scores 1,2 , and 3 . The results showed that $\log$ IMT increases approximately linearly with the fatty liver score, and also because the total sample size, 62, is small, the regressions were re-calculated using the values $0,1,2$, and 3 
Table 1. Clinical characteristics, laboratory data, and carotid IMT values in obese children with and without NAFLD and controls

\begin{tabular}{|c|c|c|c|c|c|c|}
\hline & \multirow[b]{2}{*}{ Obese children $(n=62)$} & \multirow[b]{2}{*}{ Controls $(n=30)$} & \multirow[b]{2}{*}{$p$} & \multicolumn{2}{|c|}{ Obese children } & \multirow[b]{2}{*}{$p$} \\
\hline & & & & $\begin{array}{l}\text { NAFLD } \\
(n=29)\end{array}$ & $\begin{array}{l}\text { No NAFLD } \\
(n=33)\end{array}$ & \\
\hline Age (y) & $10.0(9.4-10.7)$ & $10.1(8.8-11.3)$ & 0.89 & $10.4(9.5-11.4)$ & $10.0(9.0-11.0)$ & 0.59 \\
\hline Male, $n(\%)$ & $34(54.8)$ & 14 (46.6) & 0.50 & $15(51.7)$ & $19(57.5)$ & 0.56 \\
\hline \multicolumn{7}{|l|}{ Tanner stage, $n(\%)$} \\
\hline I & $17(27.4)$ & $10(33.3)$ & 0.16 & $7(24.1)$ & $10(30.3)$ & 0.90 \\
\hline II-III & $25(40.3)$ & $10(33.3)$ & & $13(44.8)$ & $12(36.4)$ & \\
\hline IV & $20(32.2)$ & $10(33.3)$ & & $9(31.0)$ & $11(33.3)$ & \\
\hline BMI $\left(\mathrm{kg} / \mathrm{m}^{2}\right)$ & $24.7(23.8-25.7)$ & $17.5(16.6-18.4)$ & $<0.0005$ & $25.4(24.2-26.5)$ & $24.3(23.0-25.6)$ & 0.24 \\
\hline BMI (SD score) & $1.92(1.78-2.07)$ & $-0.04(-0.39-0.29)$ & $<0.0005$ & $1.95(1.82-2.07)$ & $1.91(1.67-2.15)$ & 0.81 \\
\hline Systolic BP (mm Hg) & $113(111-115)$ & $106(104-108)$ & $<0.0005$ & $115(112-118)$ & $110(108-113)$ & 0.009 \\
\hline Diastolic BP (mm Hg) & $73(72-74)$ & $69(68-70)$ & $<0.0005$ & $74(72-76)$ & $72(71-74)$ & 0.096 \\
\hline Aspartate aminotransferase (U/L) & $30(27-33)$ & $26(24-27)$ & 0.048 & $36(31-42)$ & $26(23-28)$ & $<0.0005$ \\
\hline Alanine aminotransferase (U/L) & $35(28-42)$ & $17(15-19)$ & 0.001 & $52(39-65)$ & $22(18-27)$ & $<0.0005$ \\
\hline Total cholesterol $(\mathrm{mg} / \mathrm{dL})$ & $146(123-162)$ & $144(131-157)$ & 0.67 & $152(132-174)$ & $125(93-167)$ & 0.28 \\
\hline HDL cholesterol (mg/dL) & $38(36-40)$ & $42(40-45)$ & 0.031 & $37(34-40)$ & $39(37-42)$ & 0.31 \\
\hline LDL cholesterol (mg/dL) & $88(74-102)$ & $88(75-102)$ & 0.36 & $86(70-106)$ & $57(36-89)$ & 0.13 \\
\hline Triglycerides (mg/dL) & $105(90-123)$ & $75(65-87)$ & 0.008 & $108(91-134)$ & $99(75-130)$ & 0.48 \\
\hline Insulin $(\mu \mathrm{U} / \mathrm{mL})$ & $13.7(11.2-16.7)$ & $8.2(7.0-9.6)$ & 0.001 & $17.3(12.6-23.7)$ & $11.0(8.8-14.7)$ & 0.040 \\
\hline HOMA-IR values & $3.0(2.4-3.7)$ & $1.8(1.5-2.1)$ & 0.001 & $4.0(2.8-5.5)$ & $2.5(1.9-3.3)$ & 0.031 \\
\hline Leptin $(\mathrm{ng} / \mathrm{mL})$ & $17.0(14.6-20.0)$ & $6.0(4.8-7.5)$ & $<0.0005$ & $18.3(14.4-23.4)$ & $16.1(12.9-20.2)$ & 0.43 \\
\hline Adiponectin $(\mathrm{ng} / \mathrm{mL})$ & $11.0(9.6-12.8)$ & $12.2(10.0-14.9)$ & 0.51 & $9.3(7.4-11.7)$ & $12.7(10.6-15.1)$ & 0.029 \\
\hline Maximum IMT (mm) & $0.53(0.50-0.56)$ & $0.40(0.36-0.43)$ & $<0.0005$ & $0.58(0.54-0.62)$ & $0.49(0.46-0.52)$ & 0.001 \\
\hline Mean IMT $(\mathrm{mm})$ & $0.49(0.46-0.52)$ & $0.39(0.35-0.43)$ & $<0.0005$ & $0.54(0.50-0.58)$ & $0.45(0.42-0.48)$ & 0.001 \\
\hline
\end{tabular}

Results are expressed as $n(\%)$, mean $(95 \% \mathrm{CI})$, or geometric mean $(95 \% \mathrm{CI})$ for log-transformed variables.

Table 2. Variables independently associated with log carotid IMT in obese children

\begin{tabular}{|c|c|c|c|c|}
\hline Independent variable & Coefficient, b & $e^{b}$ & $95 \%$ CI for $\mathrm{e}^{\mathrm{b}}$ & $p$ \\
\hline \multicolumn{5}{|l|}{ Univariate linear regression analysis } \\
\hline Age (y) & 0.022 & 1.022 & $1.004-1.040$ & 0.019 \\
\hline Gender, $\mathrm{M}$ vs. F & 0.024 & 1.024 & $0.920-1.135$ & 0.65 \\
\hline Tanner stage & 0.056 & 1.057 & $1.024-1.090$ & 0.001 \\
\hline BMI $\left(\mathrm{kg} / \mathrm{m}^{2}\right)$ & 0.020 & 1.020 & $1.007-1.034$ & 0.004 \\
\hline BMI-SD score & 0.011 & 1.011 & $0.922-1.107$ & 0.81 \\
\hline Fat mass (kg) & 0.009 & 1.009 & $1.003-1.014$ & 0.005 \\
\hline Systolic BP (mm Hg) & 0.009 & 1.009 & $1.002-1.016$ & 0.009 \\
\hline Diastolic BP (mm Hg) & 0.003 & 1.003 & $0.992-1.015$ & 0.59 \\
\hline Total cholesterol $(\mathrm{mg} / \mathrm{dL})$ & 0.001 & 1.001 & $0.998-1.005$ & 0.06 \\
\hline HDL cholesterol (mg/dL) & -0.002 & 0.998 & $0.991-1.004$ & 0.47 \\
\hline LDL cholesterol (mg/dL) & 0.001 & 1.001 & $0.998-1.007$ & 0.18 \\
\hline Triglycerides (mg/dL) & 0.002 & 1.002 & $0.910-1.009$ & 0.96 \\
\hline Aspartate aminotransferase (U/L) & 0.003 & 1.003 & $0.999-1.008$ & 0.10 \\
\hline Alanine aminotransferase (U/L) & 0.003 & 1.003 & $1.001-1.004$ & 0.003 \\
\hline Insulin $(\mu \mathrm{U} / \mathrm{mL})$ & 0.004 & 1.004 & $1.002-1.007$ & 0.002 \\
\hline HOMA-IR & 0.020 & 1.020 & $1.008-1.031$ & 0.001 \\
\hline Leptin (ng/mL) & 0.003 & 1.003 & $1.001-1.007$ & 0.028 \\
\hline Adiponectin $(\mathrm{ng} / \mathrm{mL})$ & -0.009 & 0.991 & $0.983-0.999$ & 0.022 \\
\hline Fatty liver severity score & 0.081 & 1.084 & $1.041-1.128$ & $<0.0005$ \\
\hline \multicolumn{5}{|l|}{ Stepwise regression analysis* } \\
\hline Fatty liver severity score & 0.080 & 1.083 & $1.041-1.119$ & $<0.0005$ \\
\hline Tanner stage & 0.053 & 1.053 & $1.022-1.084$ & 0.001 \\
\hline
\end{tabular}

* Results of a stepwise regression analysis started with the following independent variables: age, gender, Tanner stage, BMI, fat mass, systolic and diastolic $\mathrm{BP}$, total, LDL, and HDL cholesterol, triglycerides, HOMA-IR values, adiponectin, and leptin levels.

as the measure of severity of fatty liver. In a multivariate regression model, even after adjusting for age, gender, Tanner stage, BMI, fat mass, systolic and diastolic BP, total, LDL and HDL cholesterol, triglycerides, HOMA-IR values, adiponectin and leptin levels, the severity of fatty liver was significantly associated with maximum IMT (Table 2).

\section{DISCUSSION}

This is the first study in obese children with fatty liver concerning carotid IMT values and their relationship to cardiovascular risk factors such as hypertension, dyslipidemia, insulin resistance, and adipokine levels. The present results 
demonstrate that 1) obese patients with NAFLD have a marked increase in carotid IMT than control children; 2) carotid IMT is higher for obese children with NAFLD than those with no liver involvement but with similar BMI; and 3) the severity of fatty liver is independently associated with carotid IMT after adjustment for a broad spectrum of potential confounders, including insulin resistance, a major atherogenic risk factor.

Autopsy studies in adolescents and young adults suggest that atherosclerosis begins in adolescence and that the traditional risk factors including dyslipidemia, hypertension, and diabetes are associated with its development $(20,21)$. Studies using carotid ultrasound to measure IMT in children at high risk for atherosclerotic disease are consistent with these autopsy findings. Increased IMT has been reported in children with familial hypercholesterolemia, diabetes, hypertension, and childhood obesity (22-30). Obesity in children is predictive of a wide range of health problems later in life, including an increased risk of cardiovascular morbidity and mortality. Functional and morphologic alterations of the vascular wall may therefore be expected in young obese individuals. An early study in severely obese children has reported endothelial dysfunction and increased arterial wall stiffness, but without significant intima-media thickening (26). Recent studies comparing obese and nonobese children, however, have found a significantly increased IMT (27-30). Yet, a significant reduction in carotid IMT was found among obese subjects after physical exercise and diet, suggesting a certain degree of reversibility of early atherosclerotic changes (31). The present study confirms increased carotid IMT in obese children (regardless of liver involvement). Furthermore, we have demonstrated that an association between NAFLD and increased carotid IMT is already present in children.

Recent cross-sectional studies have demonstrated a marked increase in carotid IMT in adult patients with ultrasonographically diagnosed NAFLD $(4,6)$. These results have been confirmed in a large study involving adult patients with biopsyproven NAFLD (9). The authors have shown that patients with biopsy-proven NAFLD have a significant increase in carotid IMT in comparison with age-, gender-, and BMI-matched healthy controls; carotid IMT was significantly higher for individuals with NASH than for those with simple steatosis, and, more importantly, the histologic severity of NAFLD predicted carotid IMT independently of classical risk factors, insulin resistance, and other components of the metabolic syndrome. Accumulating evidence also implies that NAFLD is correlated with circulatory endothelial dysfunction, another marker of early atherosclerosis (32). It was shown that nondiabetic NAFLD patients had a significant decrease in endothelium-dependent vasodilation compared with control subjects and that this decrease was significantly associated with the histologic severity of NAFLD, independently of age, sex, BMI, HOMA-IR, and other components of the metabolic syndrome (32).

The biologic mechanisms by which NAFLD contributes to accelerated atherosclerosis, independently of other risk factors, are still poorly understood. Several studies have documented that insulin resistance predict CVD and play a crucial role in the development of poor clinical outcome in NAFLD patients $(2,3,33)$. We found an association between insulin resistance and carotid IMT in univariate analysis that was no longer significant after adjustment for other variables. We used a surrogate estimate of insulin resistance, the HOMA score, which is well correlated with the hyperinsulinemic, euglycemic clamp technique (34). The findings suggest that hepatic steatosis may be atherogenic beyond its association with insulin resistance.

Decreased adiponectin concentrations, an adipose-secreted cytokine with antiatherogenic properties, may represent another possible underlying mechanism linking NAFLD and atherosclerosis $(35,36)$. Prospective studies have reported an association between low adiponectin levels and CVD (37). Patients with NAFLD also have a marked decrease in plasma adiponectin concentrations, and this decrease is associated with the histologic severity of NAFLD independently of abdominal obesity and other components of the metabolic syndrome (36). Thus, hypoadiponectinemia independently predicts both CVD and the metabolic syndrome (35). To date, only one study has investigated in adults the interplay among adiponectin, NAFDL, and atherosclerosis [as determined by brachial-ankle pulse wave velocity (baPWV)] (38). The authors showed that NAFLD was significantly associated with adiponectin levels and baPWV, but they did not find a significant correlation between adiponectin values and baPWV. In our group of obese children with NAFLD, adiponectin concentrations were significantly lower than those of obese children without NAFLD; however, hypoadiponectinemia was not an independent predictor of carotid IMT. Thus, it is conceivable that other atherogenic mechanisms could be involved in patients with NAFLD including enhanced oxidative stress and subclinical inflammation. Obese subjects as well as patients with NAFLD frequently have circulating markers of inflammation, and atherosclerosis is systematically associated with inflammation $(2,3,17,28,39)$. We have recently reported increased CD4-positive T cells secreting interferon- $\gamma$, a proinflammatory $\mathrm{T}$ helper 1 cytokine, in obese children (40). The increased expression of this cytokine was related to insulin resistance as well as to NAFLD independently of anthropometric features and other metabolic characteristics. Th1 cells are considered pro-atherogenic because interferon- $\gamma$ secreted by these cells decreases collagen production and activates macrophages, effects that favor plaque rupture. Further studies will assess the role and interplay of circulating molecules (including proinflammatory cytokines) on both progressive liver disease and systemic atherosclerosis in children.

Limitations of our study include its cross-sectional nature, which prevents any causal inference; the absence of liver biopsy data, which prevented us from confirming whether the severity of NAFLD can predict carotid atherosclerosis independently of other atherogenic risk factors; and the small number of subjects. No calculations of sample size requirements were made a priori. However, if the power of the study is calculated a posteriori using the observed results, it can be shown that with these sample sizes, the study had a power of 99\% to discover the observed difference between the maximum IMT in the NAFLD patients and those without NAFLD 
significantly at the 5\% level. Similarly for the comparison between the obese and controls, the power was more than $99 \%$ to discover the observed difference in maximum IMT significantly at the $5 \%$ level.

In summary, our results suggest that, in obese children, there is an independent association between NAFLD and subclinical carotid atherosclerosis. Follow-up studies are necessary to validate these findings and to determine whether this higher prevalence of atherosclerosis among children with steatosis affects long-term morbidity.

\section{REFERENCES}

1. Mager DR, Roberts EA 2006 Nonalcoholic fatty liver disease in children. Clin Liver Dis 10:109-131

2. Angulo P 2002 Nonalcoholic fatty liver disease. N Engl J Med 346:1221-1231

3. McCullough AJ 2004 The clinical features, diagnosis and natural history of nonalcoholic fatty liver disease. Clin Liver Dis 8:521-533

4. Targher G, Bertolini L, Padovani R, Zenari L, Zoppini G, Falezza G 2004 Relations of nonalcoholic hepatic steatosis to early carotid atherosclerosis in healthy men. Role of visceral fat accumulation. Diabetes Care 27:2498-2500

5. Brea A, Mosquera D, Martìn E, Arizti A, Cordero JL, Ros E 2005 Nonalcoholic fatty liver disease is associated with carotid atherosclerosis. A case-control study. Arterioscler Thromb Vasc Biol 25:1045-1050

6. Völzke H, Robinson DM, Kleine V, Deutscher R, Hoffmann W, Lüdermann J, Schminke U, Kessler C, John U 2005 Hepatic steatosis is associated with an increased risk of atherosclerosis. World J Gastroenterol 11:1848-1853

7. Lin Y-C, Lo H-M, Chen J-D 2005 Sonographic fatty liver, overweight and ischemic heart disease. World J Gastroenterol 11:4838-4842

8. Ioannou GN, Weiss NS, Boyko EJ, Mozaffarian D, Lee SP 2006 Elevated serum alanine aminotransferase activity and calculated risk of coronary heart disease in the United States. Hepatology 43:1145-1151

9. Targher G, Bertolini L, Padovani R, Rodella S, Zoppini G, Zenari L, Cigolini M, Falezza G, Arcaro G 2006 Relations between carotid artery wall thickness and liver histology in subjects with nonalcoholic fatty liver disease. Diabetes Care 29:13251330

10. Salonen JT, Salonen R 1993 Ultrasound B-mode imaging in observational studies of atherosclerosis progression. Circulation 87:II56-II65

11. Gnasso A, Irace C, Mattiolo PL, Pujia A 1996 Carotid intima-media thickness and coronary heart disease factors. Atherosclerosis 119:7-15

12. Rosfors S, Hallerstam S, Jensen-Urstad K, Zetterling M, Carlstrom C 1998 Relationship between intima-media thickness in the common carotid artery and atherosclerosis in the carotid bifurcation. Stroke 29:1378-1382

13. Raitakari OT, Juonala M, Kähönen M, Taittonen L, Laitinen T, Mäki-Torkko N, Järvisalo MJ, Uhari M, Jokinen E, Rönnemaa T, Akerblom HK, Viikari JS 2003 Cardiovascular risk factors in childhood and carotid artery intima-media thickness in adulthood: the Cardiovascular Risk in Young Finns Study. JAMA 290:2277-2283

14. De Groot E, Hovingh K, Wiegman A, Duriez P, Smit AJ, Fruchart J-C, Kastelein JJ 2004 Measurement of arterial wall thickness as a surrogate marker for atherosclerosis. Circulation 109:III33-III38

15. Marshall WA, Tanner JM 1969 Variations in pattern of pubertal changes in girls Arch Dis Child 44:291-303

16. Marshall WA, Tanner JM 1970 Variations in the pattern of pubertal changes in boys Arch Dis Child 45:13-23

17. Cole TJ, Bellizzi MC, Flegal KM, Dietz WH 2000 Establishing a standard definition for child overweight and obesity worldwide: international survey. BMJ 320:12401243

18. Pacifico L, Celestre M, Anania C, Paoloantonio P, Chiesa C, Laghi A 2007 MRI and ultrasound for hepatic fat quantification. Relationship to clinical and metabolic characteristics of pediatric nonalcoholic fatty liver disease. Acta Paediatr 96:542547
19. Hurwitz Eller N, Netterstrom B 2001 The intima media thickness and coronary risk factors. Int Angiol 20:118-125

20. Newman WP III, Freedman DS, Voors AW, Gard PD, Srinivasan SR, Cresanta JL, Williamson GD, Webber LS, Berenson GS 1986 Relation of serum lipoprotein levels and systolic blood pressure to early atherosclerosis. The Bogalusa Heart Study. N Engl J Med 314:138-144

21. Wissler RW, Strong JP 1998 Risk factors and progression of atherosclerosis in youth. PDAY Research Group. Pathological Determinants of Atherosclerosis in Youth. Am J Pathol 153:1023-1033

22. Tonstad S, Joakimsen O, Stensland-Bugge E, Leren TP, Ose L, Russel D, Bonna KH 1996 Risk factors related to carotid intima-media thickness and plaque in children with familial hypercholesterolemia and control subjects. Arterioscler Thromb Vasc Biol 16:984-991

23. Järvisalo MJ, Putto-Laurila A, Jartti L, Lehtimäki T, Solakivi T, Rönnemaa T, Raitakari OT 2002 Carotid artery intima-media thickness in children with type 1 diabetes. Diabetes 51:493-498

24. Krantz JS, Mack WJ, Hodis HN, Liu C-R, Liu C-H, Kaufman FR 2004 Early onset of subclinical atherosclerosis in young persons with type 1 diabetes. J Pediatr 145:452-457

25. Sorof JM, Alexandrov AV, Cardwell G, Portman RJ 2003 Carotid artery intimalmedial thickness and left ventricular hypertrophy in children with elevated blood pressure. Pediatrics 111:61-66

26. Tounian P, Aggoun Y, Dubern B, Varille V, Guy-Grand B, Sidi D, Girardet JP, Bonnet D 2001 Presence of increased stiffness of the common carotid artery and endothelial dysfunction in severely obese children: a prospective study. Lancet 358:1400-1404

27. Woo KS, Chook P, Yu CW, Sung RY, Qiao M, Leung SS, Lam CW, Metreweli C, Celermajer DS 2004 Overweight in children is associated with arterial endothelial dysfunction and intima-media thickening. Int J Obes Relat Metab Disord 28:852857

28. Kapiotis S, Holzer G, Schadler G, Haumer M, Widhalm H, Weghber D, Jilma B, Röggla G, Wolzt M, Widhalm K, Wagner OF 2006 A proinflammatory state is detectable in obese children and is accompanied by functional and morphological vascular changes. Arterioscler Thromb Vasc Biol 26:2541-2546

29. Atabek ME, Pirgon O, Kivrak AS 2007 Evidence for association between insulin resistance and premature carotid atherosclerosis in childhood obesity. Pediatr Res 61:345-349

30. Meyer AA, Kundt G, Steiner M, Schuff-Werner P, Kienast W 2006 Impaired flow-mediated vasodilation, carotid artery intima-media thickening, and elevated endothelial plasma markers in obese children: the impact of cardiovascular risk factors. Pediatrics 117:1560-1567

31. Wunsch R, de Sousa G, Toschke AM, Reinehr T 2006 Intima-media thickness in obese children before and after weight loss. Pediatrics 118:2334-2340

32. Villanova N, Moscatiello S, Ramilli S, Bugianesi E, Magalotti D, Vanni E, Zoli M, Marchesini G 2005 Endothelial dysfunction and cardiovascular risk profile in nonalcoholic fatty liver disease. Hepatology 42:473-480

33. Eckel RH, Grundy SM, Zimmet PZ 2005 The metabolic syndrome. Lancet $365: 1415-1428$

34. Bonora E, Targher G, Alberiche M, Bonadonna RC, Saggiani F, Zenere MB, Monauni T, Muggeo M 2000 Homeostasis model assessment closely mirrors the glucose clamp technique in the assessment of insulin sensitivity. Diabetes Care 23:57-63

35. Santaniemi M, Kesäniemi YA, Ukkola O 2006 Low plasma adiponectin concentration is an indicator of the metabolic syndrome. Eur J Endocrinol 155:745-750

36. Targher G, Bertolini L, Rodella S, Zoppini G, Scala L, Zenari L, Falezza G 2006 Associations between plasma adiponectin concentrations and liver histology in patients with nonalcoholic fatty liver disease. Clin Endocrinol (Oxf) 64:679-683

37. Pischon T, Girman CJ, Hotamisligil GS, Rifai N, Hu FB, Rimm EB 2004 Plasma adiponectin levels and risk of myocardial infarction in men. JAMA 291:1730-1737

38. Kim SG, Kim HY, Seo JA, Lee KW, Oh JH, Kim NH, Choi KM, Baik SH, Choi DS 2005 Relationship between serum adiponectin concentration, pulse wave velocity and nonalcoholic fatty liver disease. Eur J Endocrinol 152:225-231

39. Fantuzzi G, Mazzone T 2007 Adipose tissue and atherosclerosis. Exploring the connection. Arterioscler Thromb Vasc Biol 27:996-1003

40. Pacifico L, Di Renzo L, Anania C, Osborn JF, Ippoliti F, Schiavo E, Chiesa C 2006 Increased T-helper interferon- $\gamma$ - secreting cells in obese children. Eur J Endocrinol 154:691-697 